\title{
Correction to: Update of Guidelines for laparoscopic treatment of ventral and incisional abdominal wall hernias (International Endohernia Society (IEHS))—Part A
}

\author{
R. Bittner ${ }^{1,2} \cdot$ K. Bain ${ }^{3} \cdot$ V. K. Bansal ${ }^{4} \cdot$ F. Berrevoet ${ }^{5} \cdot$ J. Bingener-Casey $^{6} \cdot$ D. Chen ${ }^{7} \cdot$ J. Chen $^{8} \cdot$ P. Chowbey ${ }^{9} \cdot$

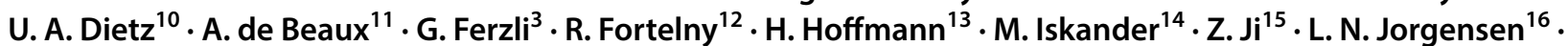

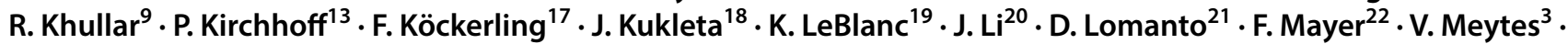 \\ M. Misra ${ }^{23} \cdot$ S. Morales-Conde ${ }^{24} \cdot$ H. Niebuhr ${ }^{25} \cdot$ D. Radvinsky ${ }^{26} \cdot$ B. Ramshaw ${ }^{27} \cdot$ D. Ranev $^{28} \cdot$ W. Reinpold ${ }^{29}$.

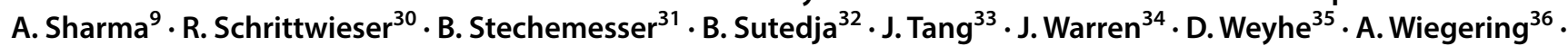 \\ G. Woeste ${ }^{37} \cdot$ Q. Yao ${ }^{38}$
}

Published online: 12 July 2019

(c) The Author(s) 2019

\section{Correction to: Surgical Endoscopy} https://doi.org/10.1007/s00464-019-06907-7

This article was updated to include the middle initial of author L. N. Jorgensen's name.

The original article can be found online at https://doi.org/10.1007/ s00464-019-06907-7.

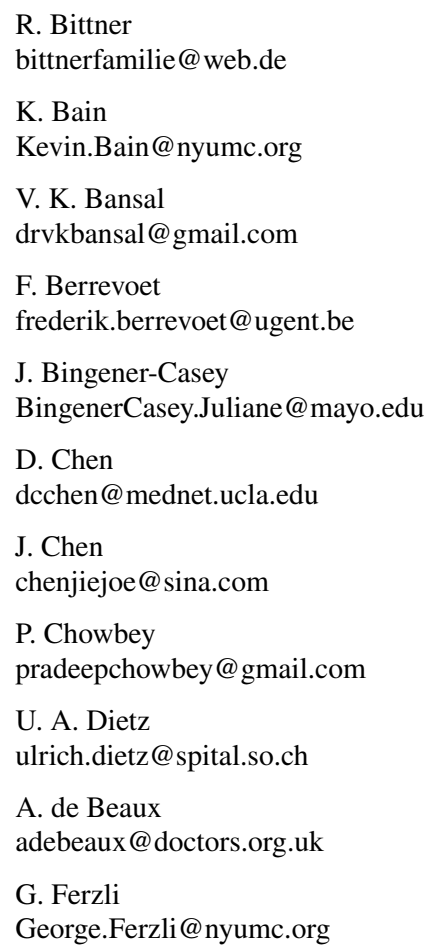

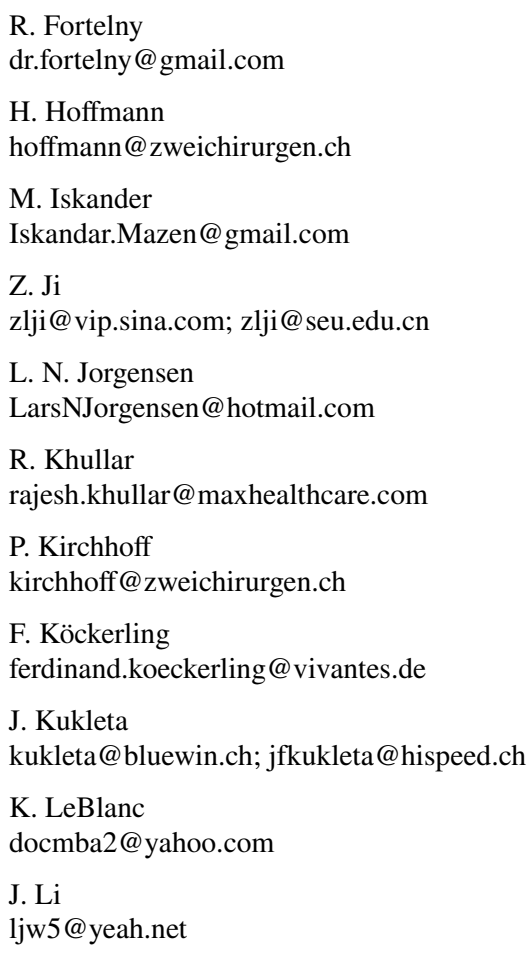


D. Lomanto

davide_lomanto@nuhs.edu.sg; surdl@nus.edu.sg

F. Mayer

f.mayer.chirurg@icloud.com

V. Meytes

vadim.meytes@gmail.com

M. Misra

info@mgumst.org

S. Morales-Conde

smoralesc@gmail.com

H. Niebuhr

henning.niebuhr@icloud.com

D. Radvinsky

dradvins@gmail.com

B. Ramshaw

bruceramshawmd@gmail.com

D. Ranev

dranev@northwell.edu

W. Reinpold

wreinpold@gmx.net

A. Sharma

Anil.Sharma@maxhealthcare.com

R. Schrittwieser

schrrudo@gmail.com

B. Stechemesser

bernd.stechemesser@hernienzentrumkoeln.de

B. Sutedja

dr.barlian@gadingpluit-hospital.com

J. Tang

johnxiong@china.com

J. Warren

JWarren1@ghs.org

D. Weyhe

dirk.weyhe@pius-hospital.de

A. Wiegering

wiegering_a@ukw.de

G. Woeste

Woeste.Guido@eke-da.de

Q. Yao

stevenyao@huashan.org.cn

1 I.M. Sechenov First Moscow State Medical University of the Ministry of Health of the Russian Federation (Sechenov University), Trubetskaya str., 8, b. 2, 119992 Moscow, Russia

2 Emeritus Director Marienhospital Stuttgart, Supperstr. 19, 70565 Stuttgart, Germany

3 Department of Surgery, New York University, New York, USA

4 Department of Surgical Disciplines, All India Institute of Medical Sciences, Room No. 5026A, 5th Floor, Teaching Block, Ansari Nagar, New Delhi 110029, India

5 Universitair Ziekenhuis Gent, C. Heymanslaan 10, 9000 Ghent, Belgium
6 Division of Breast, Endocrine, Metabolic \& Gastrointestinal Surgery, Mayo Clinic, 200 First Street SW, Rochester, MN 55905, USA

7 Lichtenstein Amid Hernia Clinic at UCLA, Section of Minimally Invasive Surgery, UCLA Division of General Surgery, Los Angeles, USA

8 Department of Hernia and Abdominal Surgery, Beijing Chao-Yang Hospital, Capital Medical University, Fengtai, China

9 Max Super Speciality Hospital, 2 Press Enclave Road, Saket, New Delhi 110017, India

10 Klinik für Viszeral-, Gefäss- und Thoraxchirurgie, Kantonsspital Olten, Baslerstrasse 150, 4600 Olten, Switzerland

11 Royal Infirmary of Edinburgh, Edinburgh EH16 4SA, UK

12 Allgemein-, Viszeral- und Tumorchirurgie, Wilhelminenspital, 1160 Vienna, Austria

13 ZweiChirurgen $\mathrm{GmbH}$, Zentrum für Hernienchirurgie und Proktologie, St. Johanns-Vorstadt 44, 4056 Basel, Switzerland

14 Department of Surgery, Mount Sinai Hospital, 1010 5th Avenue, New York, NY 10028, USA

15 Department of Surgery, Southeast University School of Medicine, Main Add. 87 Ding Jia Qiao, Nanjing 210009, Jiangsu, China

16 Digestive Disease Center, Bispebjerg Hospital, University of Copenhagen, 2400 Copenhagen NV, Denmark

17 Visceral- und Gefäßchirurgie, Zentrum für Minimal Invasive Chirurgie, Vivantes Klinikum Spandau, Neue Bergstraße 6, 13585 Berlin, Germany

18 Klinik im Park, Grossmuensterplatz 9, 8001 Zurich, Switzerland

19 Our Lady of the Lake Physician Group, 7777 Hennessy Blvd., Suite 612, Baton Rouge, LA 70808, USA

20 Department of General Surgery, Ruijin Hospital, Shanghai Jiao Tong University School of Medicine, Shanghai 200025, China

21 Department of Surgery, YLL School of Medicine, National University Hospital, Level 2, Kent Ridge Wing 2, 5 Lower Kent Ridge Road, Singapore 119074, Singapore

22 Paracelsus Medizinische Universität Salzburg (PMU), Universitätsklinik für Chirurgie, Salzburg, Austria

23 Mahatma Gandhi University of Medical Sciences \& Technology, RIICO Institutional Area, Tonk Road, Sitapura, Jaipur, Rajasthan 302 022, India

24 Centro de Cirugía Mayor Ambulatoria Ave María, Avda. de la Palmera, 53, 41013 Seville, Spain

25 HANSECHIRURGIE, Niebuhr Marleschki \& Partner, Alte Holstenstr. 16, 21031 Hamburg, Germany

26 SUNY Downstate Medical Center, 450 Clarkson Avenue, Brooklyn, NY 11203, USA

27 Department of Surgery, University Surgeons Associates, 1930 Alcoa Highway, Bldg A, Ste 285, Knoxville, TN 37920, USA 
28 Lenox Hill Hospital-Northwell Health, New York, USA

29 Abteilung für Chirurgie, Wilhelmsburger Krankenhaus, Groß-Sand 3, 21107 Hamburg, Germany

30 Abteilung für Chirurgie, LKH Hochsteiermark, Standort Bruck an der Mur Tragösser Str. 1, 8600 Bruck an der Mur, Austria

31 Hernienzentrum Köln, Zeppelinstraße 1, 50667 Cologne, Germany

32 Gading Pluit Hospital, Jl. Boulevard Timur Raya Kelapa Gading, Jakarta 14250, Indonesia

33 Department of General Surgery, Huadong Hospital, Fudan University, Shanghai, China

34 Minimally Invasive Surgery, Greenville Health System, Department of Surgery, University of South Carolina School of Medicine, Greenville, USA
35 Pius-Hospital Oldenburg, Klinik für Allgemein- und Viszeralchirurgie, Universitätsklinik für Viszeralchirurgie, Georgstraße 12, 26121 Oldenburg, Germany

36 Department of General, Visceral, Vascular and Paediatric Surgery, University Hospital of Wuerzburg, Oberduerrbacher Strasse 6, 97080 Würzburg, Germany

37 AGAPLESION ELISABETHENSTIFT gemeinnützige $\mathrm{GmbH}$, Akademisches Lehrkrankenhaus, Landgraf-Georg-Strasse 100, 64287 Darmstadt, Germany

38 Department of Hernia and Abdominal Surgery, Huashan Hospital, Fudan University, Shanghai, China 